\title{
Atomic clusters of magnetic oxides: Structure and phonons
}

\author{
A. Kirilyuk ${ }^{\mathrm{a})}$ \\ NSRIM Institute, University of Nijmegen, Toernooiveld 1, 6525 ED Nijmegen, The Netherlands \\ K. Demyk, G. von Helden, and G. Meijer \\ FOM Institute for Plasma Physics Rijnhuizen, Edisonbaan 14, 3439 MN Nieuwegein, The Netherlands
}

A. I. Poteryaev and A. I. Lichtenstein

NSRIM Institute, University of Nijmegen, Toernooiveld 1, 6525 ED Nijmegen, The Netherlands

(Presented on 13 November 2002)

This work represents a combined experimental and theoretical study of structural and magnetic properties of clusters made of cobalt, chromium, and manganese oxides. The clusters were prepared in a molecular cluster source by oxidation of laser-vaporized metal and studied in a time-of-flight spectrometer. Infrared laser-induced cluster dissociation experiments revealed the spectrum of cluster vibrational states. We also performed ab initio local spin density approximation calculations of the equilibrium geometry, electronic structure, and magnetic properties of these clusters. (C) 2003 American Institute of Physics. [DOI: 10.1063/1.1558252]

\section{INTRODUCTION}

Nanometer-sized magnetic particles have generated continuous interest since the late 1940s, as the study of their properties has proved to be scientifically and technologically very challenging. However, only in the last decade, has the emergence of new fabrication techniques led to the possibility of making small objects with the required structural and chemical qualities. The current interest in atomic clusters stems from the main observation that their properties are very different from those of the bulk. ${ }^{1}$ One of these properties is associated with the unusual stability of clusters of a certain size, commonly referred to as magic numbers. In fact, even clusters having the same composition as bulk may possess unusual structural, electronic, and magnetic properties. $^{2,3}$

Oxides of transition metals represent a rich variety of crystallographic and magnetic structures. The magnetic exchange interaction in these materials is mediated by oxygen ions thus creating a strong dependence of the resulting interaction on the coordination number. For example, chromium dioxide $\left(\mathrm{CrO}_{2}\right)$ is a ferromagnetic metal while $\mathrm{Cr}_{2} \mathrm{O}_{3}$ is an antiferromagnetic dielectric.

Strong bonding between the metal and oxygen ions (compared to the metal-metal bond) makes these materials even much more interesting with respect to cluster formation, ${ }^{4-7}$ especially in combination with various crystallographic and magnetic structures observed in the bulk. In a cluster where the balance between bulk-type bonds and the surface states plays a major role in determining the equilibrium structure, these variations are even more interesting.

\footnotetext{
a)Electronic mail: kirilyuk@sci.kun.nl
}

For some materials, larger (tens of nanometers) size grains can be produced in bulk quantities and their properties can be studied using the standard tools available for structural analysis. Complications, however, arise due to surface contamination of those grains and the unavoidable fact that those grains are produced in many different sizes. Smaller clusters containing up to several hundred atoms can be produced via laser vaporization techniques and then studied in the gas phase. The particle densities attainable are, however, usually low. Most crystallographic or spectroscopic techniques can, therefore, not be applied to those species.

In the present work, atomic clusters of magnetic oxides are made in a laser vaporization source. Their vibrational properties are studied with the help of cluster dissociation caused by resonant absorption of multiple infrared photons. Clusters of different masses are studied to follow the variation in vibrational spectra according to the cluster size. The experimental work was supported by $a b$ initio calculations. A ferromagnetic ground state for small $\mathrm{Co}-\mathrm{O}$ clusters was found, in contrast to the antiferromagnetism of bulk $\mathrm{CoO}$.

\section{EXPERIMENTAL DETAILS}

The experimental setup was described previously ${ }^{8}$ so only a brief outline is given here. Clusters are produced by pulsed Nd:YAG laser vaporization of a metal rod at $532 \mathrm{~nm}$. A mixture of $\mathrm{O}_{2}$ in argon (or pure oxygen) quenches the plasma, clusters form, and the gas expands into the vacuum. The beam is skimmed and, if necessary, charged clusters are deflected out of the beam by static electric fields. The clusters then enter the region of interaction with the ionizing ArF laser (193 nm wavelength) situated between plates of a timeof-flight (TOF) mass spectrometer. The plates of the TOF spectrometer are initially at ground potential and are pulsed 


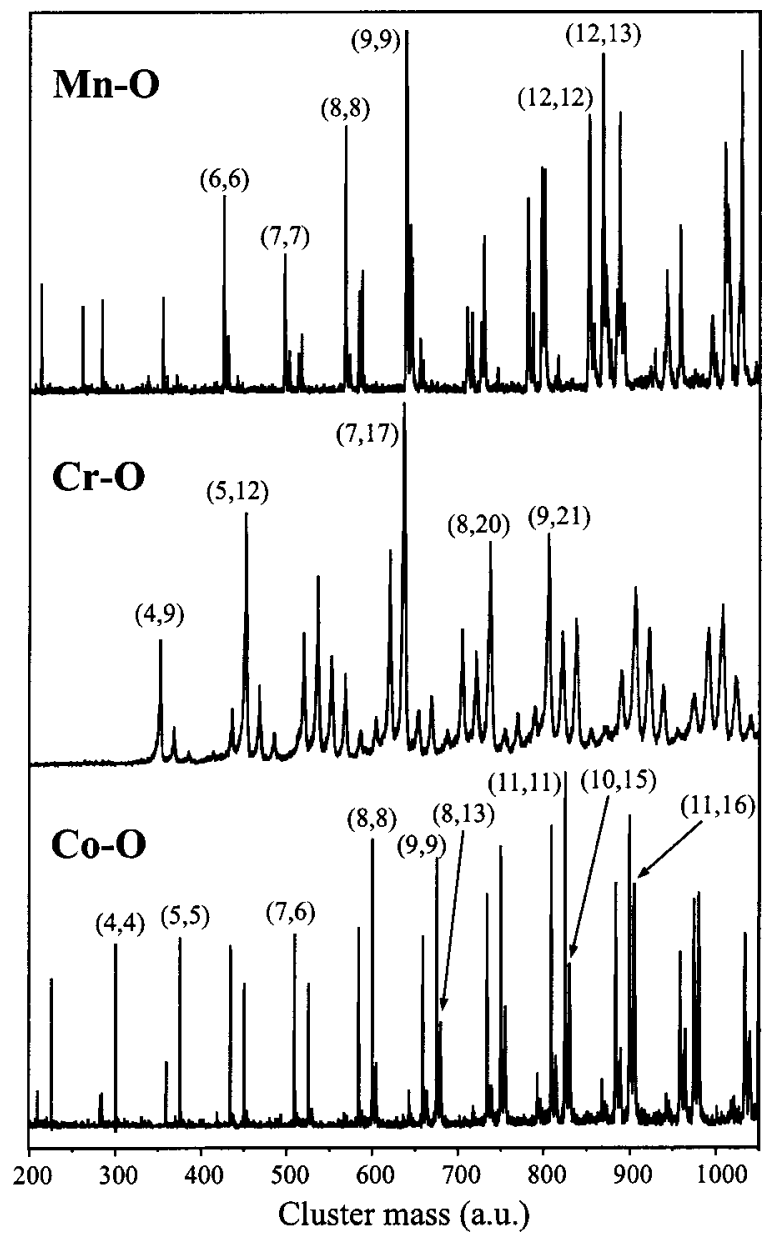

FIG. 1. Mass spectra of $\mathrm{Co}-\mathrm{O}, \mathrm{Cr}-\mathrm{O}$, and $\mathrm{Mn}-\mathrm{O}$ clusters. The composition of the most prominent peaks is indicated by $(m, n)$ for the $\mathrm{M}_{m} \mathrm{O}_{n}$ formula.

to high voltage simultaneously with the ArF laser pulse. The ions produced during that time are accelerated into the TOF spectrometer and a mass spectrum is obtained. To measure the vibrational spectra, an infrared (IR) laser is used, either in addition to the ArF laser or independently. The IR laser is the free electron laser for infrared experiments (FELIX) ${ }^{9}$ in Nieuwegein, The Netherlands. The light output of FELIX is tunable from 40 to $2000 \mathrm{~cm}^{-1}$ and consists of $(5 \mathrm{~Hz}) \mathrm{mac}-$ ropulses of $5 \mu$ s duration. Each macropulse consists of 0.3-5 ps long light pulses, spaced by $1 \mathrm{~ns}$. The bandwidth is nearly Fourier transform limited. Recording mass spectra as a function of the FELIX wavelength yields IR spectra of the individual clusters via the depletion or appearance of certain peaks in the mass spectra.

\section{RESULTS}

Figure 1 shows the mass spectra obtained for three different cluster compositions. Among them, the $\mathrm{Cr}-\mathrm{O}$ clusters could not be ionized even with focused ArF laser radiation. Therefore, ions formed in the cluster source were studied directly. The observed distribution of clusters on their composition was strongly dependent on the conditions in the cluster source, such as the back gas pressure and concentration of $\mathrm{O}_{2}$, YAG laser power, etc. In addition, excessive

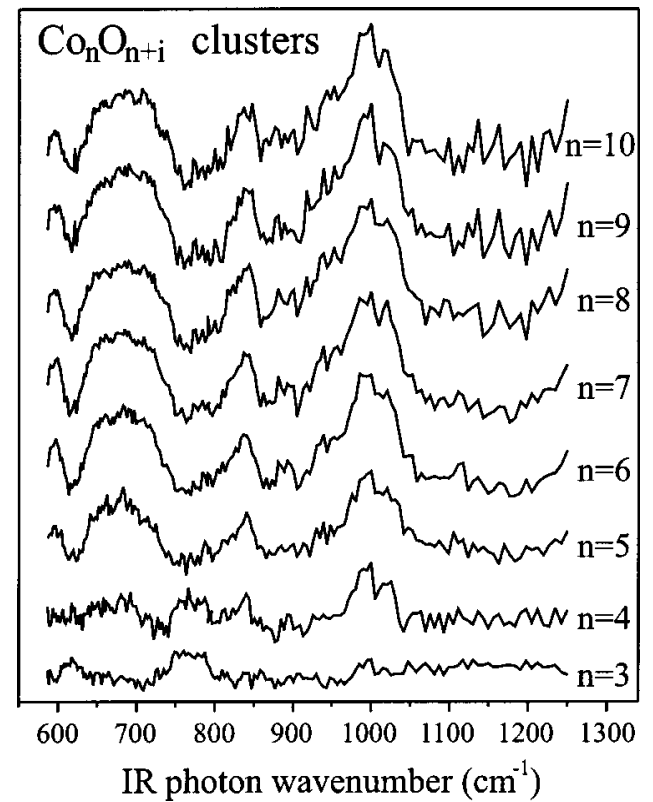

FIG. 2. Vibrational spectra of $\mathrm{Co}-\mathrm{O}$ clusters with various compositions with the general formula $\mathrm{Co}_{n} \mathrm{O}_{n+i} . n$ varies between 3 and 10 as indicated. For each $n$, summation of peaks with different $i=1-5$ was done.

focusing of the ionizing laser leads to cluster dissociation, apart from ionizing them. For the best results, the ionizing laser power should be carefully balanced.

In the mass spectra of Fig. 1, only clusters with a particular composition are observed. This could be due to a variety of factors: (a) our cluster source could be producing only species with such a stoichiometry, (b) their absorption cross section might be significantly higher, (c) they could be much more stable than clusters with other compositions, or (d) their ionization potential could be smaller than that of other clusters. Option (d) seems to be the most likely possibility. The ionization potentials of these clusters are not known.

With this in mind, it is nevertheless interesting to note that $\mathrm{Co}-\mathrm{O}$ clusters exist in two distinctive compositions: stoichiometric $\mathrm{Co}_{n} \mathrm{O}_{n}$ and with extra oxygen $\mathrm{Co}_{n} \mathrm{O}_{n+5}$. Mn-O clusters seem to exist in the stoichiometric form $\mathrm{Mn}_{n} \mathrm{O}_{n}$ only, although some heavy masses have not been identified yet. As for $\mathrm{Cr}-\mathrm{O}$, only clusters with large oxidation numbers have been observed, such as $\mathrm{Cr}_{n} \mathrm{O}_{2 n+x}, x>1$.

To obtain structural information on the clusters generated, tunable infrared laser FELIX was employed. The experiment could be done in two different modes: IR radiation is used (i) to resonantly ionize clusters or (ii) to dissociate them. In both cases, excitation of the clusters happens resonantly when the IR photon energy coincides with one of the vibrational modes of a cluster (i.e., phonons, in other words). In the dissociation experiments, one detects the depletion of one or another peak in the mass spectrum as a function of the IR wavelength. This depletion spectrum is thus a replication of the (IR-allowed) vibrational spectrum of a given cluster.

One should realize, however, that both dissociation and ionization potentials of a cluster are roughly two orders of magnitude higher than typical vibrational transitions (roughly $10 \mathrm{eV}$ compared to $0.05-0.15 \mathrm{eV}$ ). This implies an 

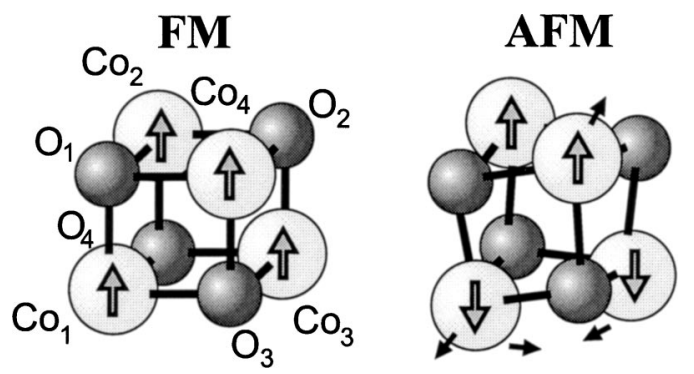

FIG. 3. Ferromagnetic vs antiferromagnetic cluster configuration. The nontetrahedral distortions of the latter are exaggerated for clarity. Small arrows show the directions of distortion.

essentially multiple-photon process. Due to unavoidable anharmonicity, the vibrational levels are not equidistant, making it difficult to observe such a process. The subpicosecond pulses and therefore wide (Fourier-limited) spectrum of FELIX make such an experiment possible.

Figure 2 shows measured spectra for $\mathrm{Co}-\mathrm{O}$ clusters of different mass. Note that the distribution of clusters in these measurements is slightly different from that shown in Fig. 1. This is due to the different experimental configuration where cluster ions from the source were directly excited with FELIX. Qualitatively, all these spectra are very similar, except the lightest ones. The visible differences can be ascribed to different excitation probabilities as well as to the fact that some part of the lighter cluster population comes as a result of dissociation of the heavier one, thus partially compensating for resonant effects.

The only phonon mode that appears in the highresolution electron energy loss spectrum has an energy of $69.4 \mathrm{meV},{ }^{10}$ corresponding to $560 \mathrm{~cm}^{-1}$. Our dissociation spectrum did not reveal any peculiarities in that region, and this might also be due to the reduced FELIX performance in that region. The observed multiple lines, on the other hand, show the low degree of symmetry of the clusters studied in comparison to those in the cubic bulk structure.

\section{AB INITIO CALCULATIONS}

We also performed $a b$ initio calculations of the equilibrium geometry and electronic structure of magnetic cluster $\mathrm{Co}_{4} \mathrm{O}_{4}$ using a VASP package. ${ }^{11}$ In the optimization, the cluster is placed in a cubic cell with an edge of $12.37 \AA$, which is enough to make dispersion between clusters negligible. We optimized the symmetry without any restrictions and a $\Gamma$ point only can be used to represent the Brillouin zone. In order to obtain good accuracy in structure optimization an energy cutoff of $500 \mathrm{eV}$ for plane-wave expansion of the pseudo wave functions was used. Optimization is terminated when forces that act on atoms are smaller than $0.015 \mathrm{eV} / \AA$. The $4 s, 4 p$, and $3 d$ orbitals for Co and $2 s, 2 p$ orbitals for the $\mathrm{O}$ atoms are used as a basis set.

We have found that the $\mathrm{Co}_{4} \mathrm{O}_{4}$ cluster has a geometry similar to that of the structure of a solid crystal with cobalt and oxygen tetrahedra intercalated with each other. The distances and total energy for ferromagnetic (FM) and antiferromagnetic (AFM) configurations, sketched in Fig. 3, are presented in Table I.
TABLE I. Calculated structure and total energy of a $\mathrm{Co}_{4} \mathrm{O}_{4}$ cluster.

\begin{tabular}{lcc}
\hline \hline & \multicolumn{2}{c}{ Interatomic distance $(\AA)$} \\
\cline { 2 - 3 } & FM & AFM \\
\hline $\mathrm{Co}_{1}-\mathrm{Co}_{2}$ & 2.366 & 2.485 \\
$\mathrm{Co}_{1}-\mathrm{Co}_{3}$ & 2.366 & 2.397 \\
$\mathrm{Co}_{1}-\mathrm{Co}_{4}$ & 2.366 & 2.485 \\
$\mathrm{Co}_{1}-\mathrm{O}_{1}$ & 1.943 & 1.907 \\
$\mathrm{Co}_{1}-\mathrm{O}_{2}$ & 1.943 & 1.941 \\
$\mathrm{Co}_{1}-\mathrm{O}_{3}$ & 1.943 & 1.907 \\
$\mathrm{Co}_{1}-\mathrm{O}_{2}$ & 3.313 & 3.297 \\
$\mathrm{O}_{1}-\mathrm{O}_{2}$ & 3.046 & 2.912 \\
$\mathrm{O}_{1}-\mathrm{O}_{3}$ & 3.046 & 2.961 \\
$\mathrm{O}_{1}-\mathrm{O}_{4}$ & 3.046 & 2.912 \\
$\mathrm{Total}_{2}$ energy $(\mathrm{eV})$ & -46.470407 & -46.291445 \\
\hline \hline
\end{tabular}

From Table I we can see that the tetrahedra for the FM cluster are ideal and the symmetry is $T_{3 d}$. The distance between all cobalt and oxygen atoms are equal. For the AFM cluster the size of the clusters is slightly smaller and the geometry of the clusters is not ideal. The symmetry of the AFM cluster is $C_{2 v}$. The distance between atoms with the same spin direction is smaller than for the AFM coupled atoms, both for cobalt and for oxygen tetrahedra (note that the oxygen atoms also possess induced magnetic moments). The ferromagnetic ground state was found, with the difference in energy of almost $200 \mathrm{meV}$ being due to structural distortion, which in turn is caused by the AFM spin arrangement.

\section{ACKNOWLEDGMENTS}

The authors gratefully acknowledge support by the Stichting voor Fundamenteel Onderzoek der Materie (FOM) in providing the required beam time on FELIX and greatly appreciate the skillful assistance of the FELIX staff, in particular Dr. A. F. G. van der Meer. Part of this work was supported by the European IST network SPINOSA (Contract No. IST-2001-33334).

${ }^{1}$ Science 271, 920 (1996).

${ }^{2}$ S. K. Nayak and P. Jena, Phys. Rev. Lett. 81, 2970 (1998).

${ }^{3}$ B. V. Reddy and S. N. Khanna, Phys. Rev. Lett. 83, 3170 (1999).

${ }^{4}$ P. J. Ziemann and A. W. Castleman, Jr., Phys. Rev. B 46, 13480 (1992).

${ }^{5}$ Q. Wang, Q. Sun, M. Sakurai, J. Z. Yu, B. L. Gu, K. Sumiyama, and Y. Kawazoe, Phys. Rev. B 59, 12672 (1999); Q. Sun, Q. Wang, K. Parlinski,

J. Z. Yu, Y. Hashi, X. G. Gong, and Y. Kawazoe, ibid. 61, 5781 (2000).

${ }^{6}$ J. Kortus and M. R. Pederson, Phys. Rev. B 62, 5755 (2000).

${ }^{7}$ Q. Sun, M. Sakurai, Q. Wang, J. Z. Yu, G. H. Wang, K. Sumiyama, and Y. Kawazoe, Phys. Rev. B 62, 8500 (2000).

${ }^{8}$ D. van Heijnsbergen, G. von Helden, M. A. Duncan, A. J. A. van Roij, and G. Meijer, Phys. Rev. Lett. 83, 4983 (1999).

${ }^{9}$ G. M. H. Knippels, R. F. X. A. M. Mols, A. F. G. van der Meer, D. Oepts, and P. W. van Amersfoort, Phys. Rev. Lett. 75, 1755 (1995).

${ }^{10}$ M. H. Nassir and M. A. Langell, Solid State Commun. 92, 791 (1994); G. A. Carson, M. H. Nassir, and M. A. Langell, J. Vac. Sci. Technol. A 14, 1637 (1996).

${ }^{11}$ G. Kresse and J. Hafner, Phys. Rev. B 47, 558 (1993); 49, 14251 (1994); G. Kresse and J. Furthmüller, ibid. 54, 11169 (1996). 